\title{
Development of a novel rat model of lumbar facet joint osteoarthritis induced by persistent compressive injury
}

\author{
YOU LU ${ }^{1,2}$, SHISHEN PEI ${ }^{3}$ and SHUXUN HOU ${ }^{1,4}$ \\ ${ }^{1}$ Department of Orthopaedics, Chinese PLA Medical School, Beijing 100853; ${ }^{2}$ Department of \\ Orthopaedics, Sixth Medical Center of PLA General Hospital, Beijing 100048; ${ }^{3}$ Department of \\ Orthopaedics, The Fourth People's Hospital of Hengshui City, Hengshui, Hebei 053000; ${ }^{4}$ Department of \\ Orthopaedics, Fourth Medical Center of PLA General Hospital, Beijing 100048, P.R. China
}

Received June 13, 2019; Accepted March 6, 2020

DOI: 10.3892/etm.2020.9117

\begin{abstract}
The aim of the present study was to develop a novel animal model of lumbar facet joint osteoarthritis induced by persistent compressive injury. An intraspinal compression spring was randomly implanted into either the L4/5 or the L5/6 spinal segments of 40 Sprague Dawley (SD) rats to induce compression. Sham-operations were used in the other segment of the same rats as the control levels. The animals were sacrificed at 7, 14, 28, 42 and 56 days after surgery, prior to the radiological confirmation of the spring location. Degeneration of the lumbar facet joints was evaluated by macroscopic observation in addition to histological and immunohistological analyses. The results of this present study revealed the absence of spring dislocation during the entire observation period. Macroscopic and Osteoarthritis Research Society International scores of the compression levels were found to be higher in the compression levels compared with those noted in the control levels $(\mathrm{P}<0.05)$. In addition, interleukin- $1 \beta$ and tumor necrosis factor- $\alpha$ expression in the compression levels were increased over time compared with those recorded in the control levels. In conclusion, persistent compressive injury may induce degeneration of the lumbar facet joint. This novel animal model could serve as a useful tool for further studies into the mechanisms of action and potential treatment of lumbar facet joint osteoarthritis.
\end{abstract}

\section{Introduction}

Lower back pain is a ubiquitous disorder that is associated with limitations to daily activities that significantly reduce

Correspondence to: Professor Shuxun Hou, Department of Orthopaedics, Chinese PLA Medical School, 28 Fuxing Road, Beijing 100853, P.R. China

E-mail: hsxortho@hotmail.com

Key words: lumbar facet joint, animal model, persisting compressive injury, rat, osteoarthritis the quality of life. Its lifetime prevalence is $>80 \%$ worldwide (1-4). Symptomatic degenerative defects to the lumbar spine are the main causes of lower back pain $(5,6)$, which costs over $\$ 100$ billion each year in the US (7). Since the process of degeneration itself does not always result in pain, factors and mechanisms that are available to distinguish symptomatic from asymptomatic degeneration remain elusive (8). Furthermore, lower back pain is not caused by a known etiology or mechanism. The origin of tissue pain may emanate from $\geq 1$ sources, including the intervertebral disc, facet joints, longitudinal ligaments, musculature and fascia $(9,10)$.

Animal models have provided valuable insights into the mechanism of symptomatic degeneration of the spine. A number of disc degeneration animal models have been established previously, which are important for mechanistic studies and the development of treatment strategies (11). However, the number of animal models of lumbar facet joint degeneration that have been established remain insufficient. As the only true synovial joints of the spine, facet joints are highly innervated and as such are important load-bearing structures in the posterior aspect of the vertebral column (12). Stress overload may result in osteoarthritis of the facet joint, which has been determined to be a possible source of lower back pain (13). Despite its prevalence, few studies have utilized animal models to study lumbar facet joint osteoarthritis. Difficulties in creating animal models stem from the fact that both chemical and mechanical interventions are more difficult to perform in the facet joint compared with the same procedures in the disc. Among these models, the majority belong to the class of chemically induced model of facet joint osteoarthritis, which induces a rapid and severe autoimmune reaction by injecting chemical agents into the lumbar facet joints (14).

In this present study, a novel rat model of mechanically-induced lumbar facet joint injury was developed. In contrast to previously described chemically-induced animal models, the current model was created by physically loading increased stress, which was closer to the etiology of human facet joint syndrome (12). This rat model may provide an ideal experimental model for further investigations into the etiology, pathogenesis and development of therapies for lumbar facet joint osteoarthritis. 


\section{Materials and methods}

Ethics. All experimental procedures were reviewed and approved by the Sixth Medical Center of the Chinese PLA General Hospital Animal Ethics Board (Beijing, China).

Design and manufacture of the compression spring. A modified intraspinal compression spring was designed according to the anatomy measurements of the male Sprague Dawley (SD) rat lumbar spine (Fig. 1). The modified intraspinal compression spring can provide persistent compressive stress to the lumbar facet joints. The spring was manufactured by the Yongxing Kangli Hardware Factory (Wenzhou, China) with the following dimensions: i) External diameter, $2 \mathrm{~mm}$; ii) length, $2 \mathrm{~mm}$; iii) wire diameter, $0.4 \mathrm{~mm}$; and iv) $2 \mathrm{~mm}$ accessory length. The string was made of $316 \mathrm{~L}$ stainless steel, which can offer $50 \mathrm{~g}$ in force when stretched to $4 \mathrm{~mm}$. The force of the spring was set according to the biomechanics of the facet joint in humans. Generally, the bilateral facet joints can bear $20 \%$ of the body weight (15). Therefore, for a rat weighing $\sim 250 \mathrm{~g}$, the application of $50 \mathrm{~g}$ in pressure can simulate the facet stress that is typically observed in humans (15).

Animal model induction. A total of 40 healthy male SD rats (aged 6-8 weeks; weight, 255-320 g) were provided by the Experimental Animal Center of the Sixth Medical Center of Chinese PLA General Hospital [Animal production license number: SCXK (Beijing) 2015-0012]. Animals were kept in cages with the environmental temperature, maintained at a temperature of $22-26^{\circ} \mathrm{C}$ and a relative humidity at $40-80 \%$ under a $12 \mathrm{~h}$ light-dark cycle, and allowed free access to food and water. Routine cleaning and disinfection were performed daily. For each rat, one of the L4/5 and L5/6 spinal fragments was selected randomly using a random number table marked as Compression level for persistent compressive injury. The other level of the same animal was indicated as Control level treated with sham operation. All rats were anesthetized by an intraperitoneal injection of ketamine $(5 \mathrm{mg} / 100 \mathrm{~g})$, xylazine $(0.5 \mathrm{mg} / 100 \mathrm{~g})$ and acepromazine $(0.1 \mathrm{mg} / 100 \mathrm{~g})$. Following skin preparation and disinfection, a posterior midline incision was performed, where the multifidus muscle at the L4-L6 spinous process was resected to expose the L4-L6 spinal processes. The modified intraspinal compression springs were implanted into the defined compression level by attachment to the spinous processes (Fig. 2). The control level in the same animal underwent the same incision and exposure as that of compression level without implantation of the compression spring. Subsequently, the muscle was sutured and the skin was then closed at both compression and control levels. The animals were treated with subcutaneous injections of antibiotics (penicillin, 80,000 Units) once per day per rat for 3 days. Following surgery, the rats were allowed to recover and were subsequently examined on days 7, 14, 28, 42 and 56 as described below.

Macroscopic observation. On days 7, 14, 28, 42 and 56 following surgery, 8 rats were randomly euthanized by an intraperitoneal overdose injection of pentobarbital sodium $(200 \mathrm{mg} / \mathrm{kg})$. Anteroposterior (AP) and lateral X-ray examinations of the rats were performed to verify the spring

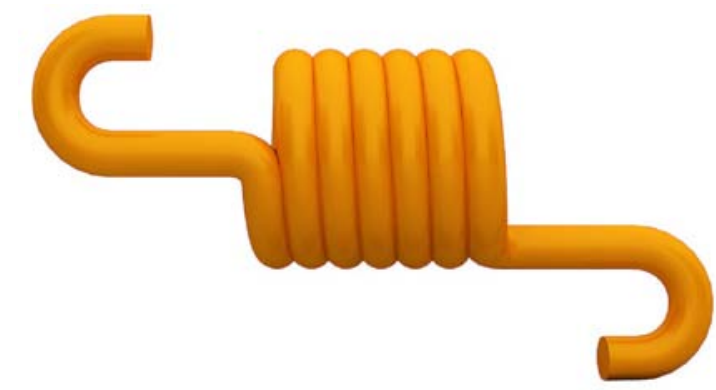

Figure 1. A modified intraspinal compression spring was manufactured by the Yongxing Kangli Hardware Factory (external diameter, $2 \mathrm{~mm}$; length, $2 \mathrm{~mm}$; wire diameter, $0.4 \mathrm{~mm}$; accessory length, $2 \mathrm{~mm}$ ).

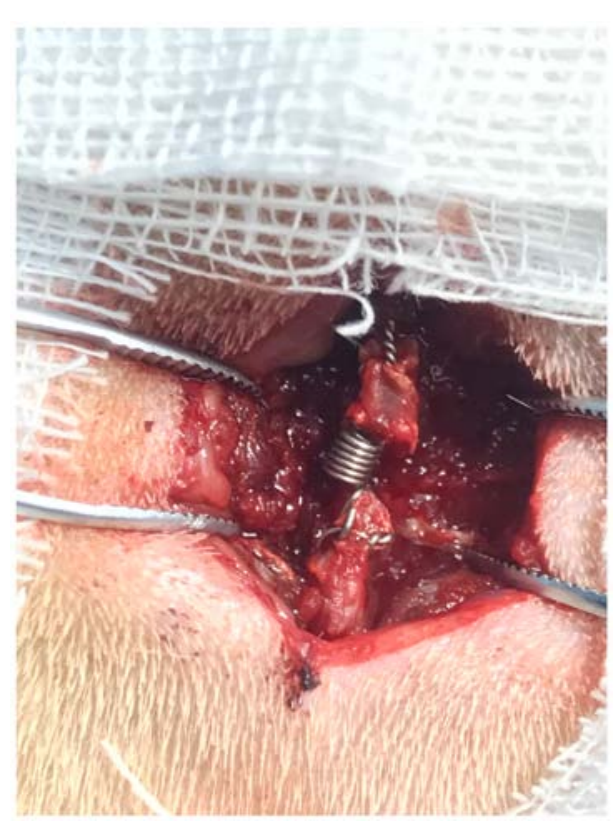

Figure 2. A representative image of the procedure demonstrating the modified intraspinal compression springs being fixed between the two spinous processes.

location prior to lumbar vertebral segment collection. The L4-L6 spinal sample was harvested en bloc after being sacrificed via cervical dislocation. The pathological defects in the L4/5 and L5/6 lumbar facet joints were evaluated by macroscopic observation. A macroscopic tissue observation scoring system proposed by Pelletier et al (16) was used, with a score of 0-4 corresponding to the following conditions: i) 0 , intact articular surface with normal calamine blue color; ii) 1, rough articular surface with a gray color; iii) 2, defected superficial cartilage; iv) 3, defected deep cartilage with ulceration; and v) 4, exfoliated cartilage with exposed subchondral bone.

Histological examination. Following macroscopic observation, the L4-L6 segments were fixed in 4\% formaldehyde for $48 \mathrm{~h}$ at room temperature, followed by decalcification in EDTA solution for 4-8 weeks. The decalcified facet joints were paraffin-embedded and subsequently sliced into 4-6 $\mu \mathrm{m}$ thick sections in the transverse plane. Serial facet joint sections of the experimental and control levels were deparaffinized using xylene followed by a descending series of ethanol at room 

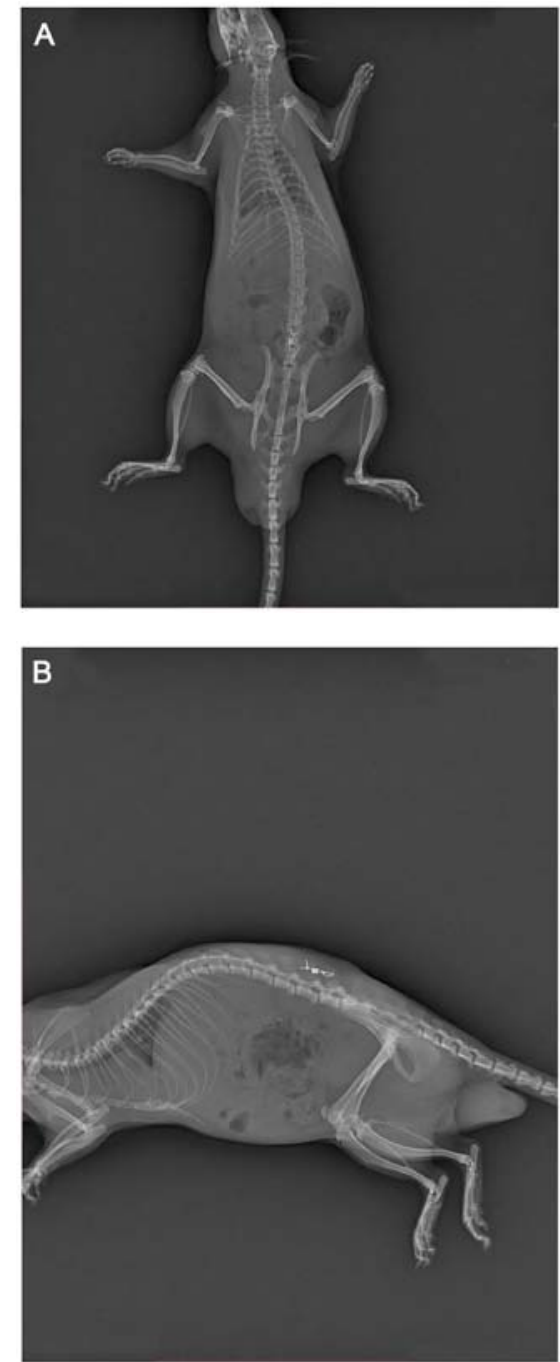

Figure 3. Representative X-ray images obtained following euthanasia of the rats, confirming that no spring dislocations were found. (A) Anteroposterior view revealing that the compression spring was located in the central of lumbar spine without dislocation. (B) Lateral view revealing that the compression spring was fastened between the adjacent spinous processes.

temperature. The sections were stained with toluidine blue at $20^{\circ} \mathrm{C}$ for $10 \mathrm{~min}$. The results were detected using a light microscope (Olympus Corporation) at a magnification of x100, and three fields of view were scored for articular cartilage degeneration according to the Osteoarthritis Research Society International (OARSI) score (ranging from 0 to 24 points) (17).

Immunohistochemical staining. The aforementioned deparaffinized facet joint sections were incubated with rabbit-anti rat antigen monoclonal antibodies targeting interleukin (IL)- $1 \beta$ (1:100; cat. no. ab9722; Abcam) and tumor necrosis factor (TNF)- $\alpha$ (1:100; cat. no. ab6671; Abcam) at $4^{\circ} \mathrm{C}$ overnight. Subsequently, the sections were further incubated with a biotin-labeled goat-anti rabbit secondary antibody (1:200; cat. no. 074-1506; KPL, Inc.) for $60 \mathrm{~min}$ at room temperature. The staining was made visible using 3,3'-diaminobenzidine, where cells exhibiting positive staining are brown in color. Staining intensity and the percentage of positive cells were evaluated from three random fields for each section under a light microscope (Olympus Corporation; magnification, x100).

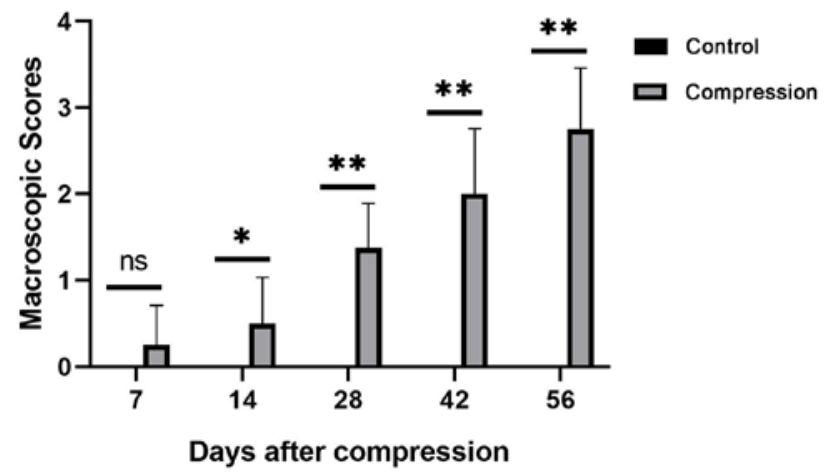

Figure 4. Comparison of macroscopic scores of the facet joint cartilage between control and compression levels 7, 14, 28, 42 and 56 days after surgery. All data are presented as the mean \pm standard deviation $(n=8$ per time point). ${ }^{*} \mathrm{P}<0.05$ and ${ }^{* *} \mathrm{P}<0.01$. ns, not significant.

A computer interface (Image-Pro Plus; version 6.0; Media Cybernetics Inc.) was used for image analysis. The relative expression of the targeted molecules was calculated by multiplying the staining intensity score with the positive cell score. Staining intensity was scored from 0 to 3, representing no staining, light, moderate and strong staining, respectively. Positive cells (the ratio of brown cells to total cells) were scored from 1 to 4 as follows: i) <10\%; ii) $11-50 \%$; iii) 51-75\%; and iv) $>75 \%$, respectively.

Statistical analysis. Continuous variables are presented as the mean $\pm \mathrm{SD}$. Comparisons made for non-parametric data, between the control and treated groups at different time points were performed using Wilcoxon signed rank sum tests with Bonferroni's adjustments and parametric data comparisons were performed using the paired Student's t-test. All statistical analyses were performed using the SPSS 17.0 software (SPSS, Inc.). $\mathrm{P}<0.05$ was considered to indicate a statistically significant difference.

\section{Results}

Macroscopic observation. AP and lateral X-ray examinations were performed on all rats at the indicated days prior to sacrifice. All springs were found to be in the correct position without any dislocation (Fig. 3). The facet joint articular cartilage in the control group exhibited a bright calamine blue color without cracks or defects at 7, 14, 28, 42 and 56 days following surgery. Compared with the control group, time-dependent pathological changes were observed in the experimental group. In addition, joint synovial membranes were slightly swollen at 7 and 14 days following surgery. Marked joint swelling and a gray, dim articular cartilage surface was observed at 28 days, whereas at 42 and 56 days following surgery, a rough cartilage surface and minor cracks were observed, respectively (data not shown).

The macroscopic scores of the facet joint cartilage at each measured day following surgery are presented in Fig. 4. Comparisons between the control and the compression groups were performed at the indicated days. Statistically significant differences were observed on days 14, 28, 42 and 56 following surgery $(\mathrm{P}<0.05$ for day 14 and $\mathrm{P}<0.01$ for all other cases). 

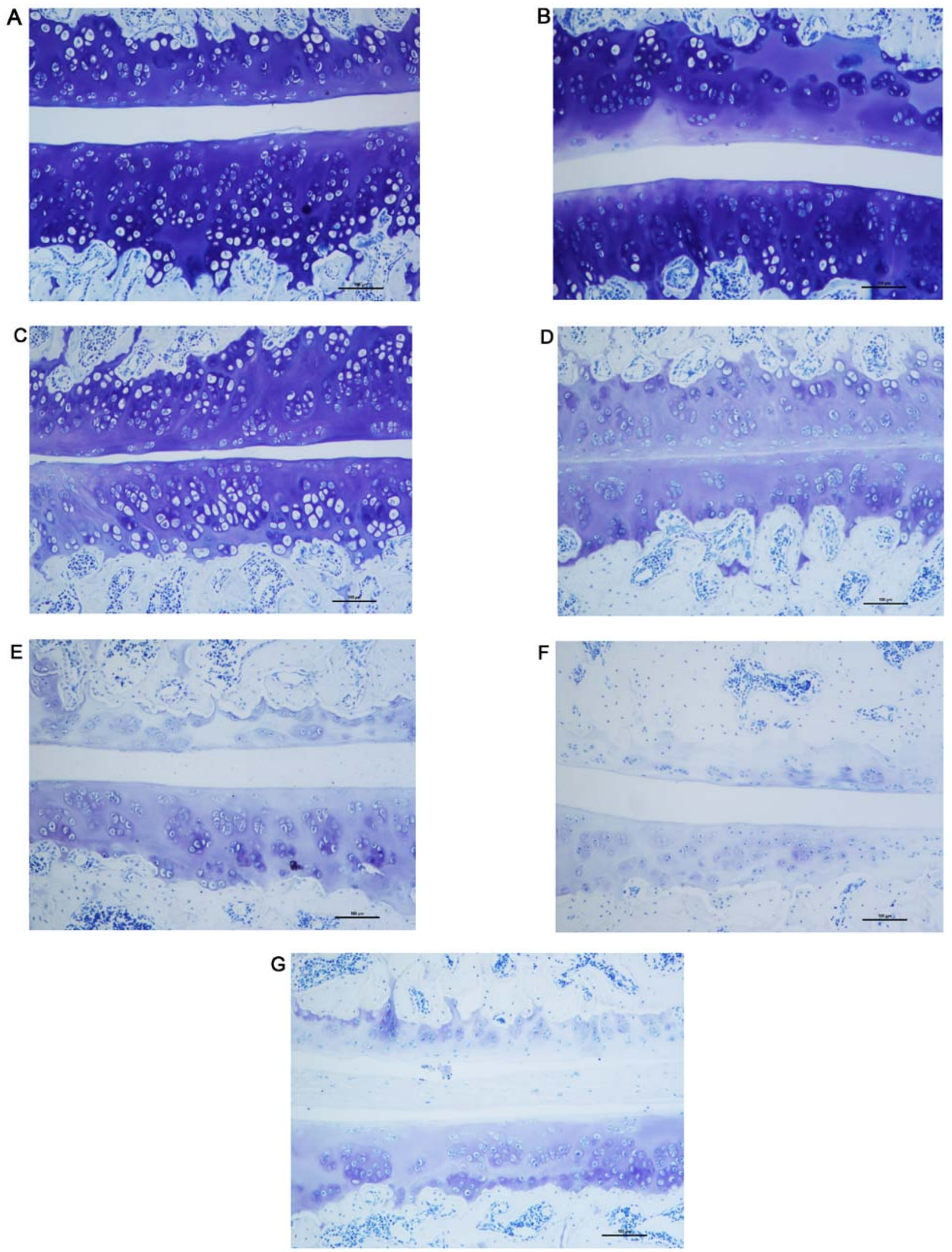

Figure 5. Histological examination of the facet joint cartilage of L4-L6 segments. Photomicrographs of facet joint cartilages in the control levels stained with toluidine blue at (A) 7 and (B) 56 days following surgery. Photomicrographs of facet joint cartilages in the compression levels stained with toluidine blue at (C) 7, (D) 14, (E) 28, (F) 42 and (G) 56 days following surgery (magnification, x100; scale bar, $100 \mu \mathrm{m}$ ).

However, significant differences were not observed at 7 days following surgery $(\mathrm{P}>0.05)$. The results suggest that after 7 days, the macroscopic scores of the facet joint cartilage in the compression levels were significantly higher compared with those in the control levels throughout the testing period $(\mathrm{P}<0.05)$. 
Table I. Immunohistochemical scores of IL-1 $\beta$ expression in cartilage in the two levels.

\begin{tabular}{llllll}
\hline Group & \multicolumn{1}{c}{7 days } & 14 days & 28 days & 42 days & 56 days \\
\hline Control level & $0.37 \pm 0.34$ & $0.54 \pm 0.47$ & $0.51 \pm 0.55$ & $0.69 \pm 0.72$ & $0.63 \pm 0.19$ \\
Compression level & $1.76 \pm 1.03^{\mathrm{a}}$ & $3.32 \pm 1.29^{\mathrm{a}}$ & $5.14 \pm 2.37^{\mathrm{a}}$ & $8.14 \pm 2.25^{\mathrm{a}}$ & $8.23 \pm 1.36^{\mathrm{a}}$ \\
T-value & 5.43 & 7.78 & 10.34 & 13.49 & 15.31 \\
\hline
\end{tabular}

${ }^{\mathrm{a}} \mathrm{P}<0.01$ vs. Control level ( $\mathrm{n}=8$ per time point).

Table II. Immunohistochemical scores of TNF- $\alpha$ expression in cartilage in the two levels.

\begin{tabular}{llllll}
\hline Group & \multicolumn{1}{c}{7 days } & 14 days & 28 days & 42 days & 56 days \\
\hline Control level & $0.45 \pm 0.38$ & $0.59 \pm 0.83$ & $0.66 \pm 0.35$ & $0.50 \pm 0.71$ & $0.63 \pm 1.09$ \\
Compression level & $1.39 \pm 0.76^{\mathrm{a}}$ & $4.03 \pm 1.99^{\mathrm{a}}$ & $4.51 \pm 3.13^{\mathrm{a}}$ & $7.36 \pm 3.14^{\mathrm{a}}$ & $8.98 \pm 2.21^{\mathrm{a}}$ \\
T-value & 3.98 & 6.95 & 7.66 & 9.93 & 14.96 \\
\hline
\end{tabular}

${ }^{\mathrm{a}} \mathrm{P}<0.01$ vs. Control level ( $\mathrm{n}=8$ per time point).

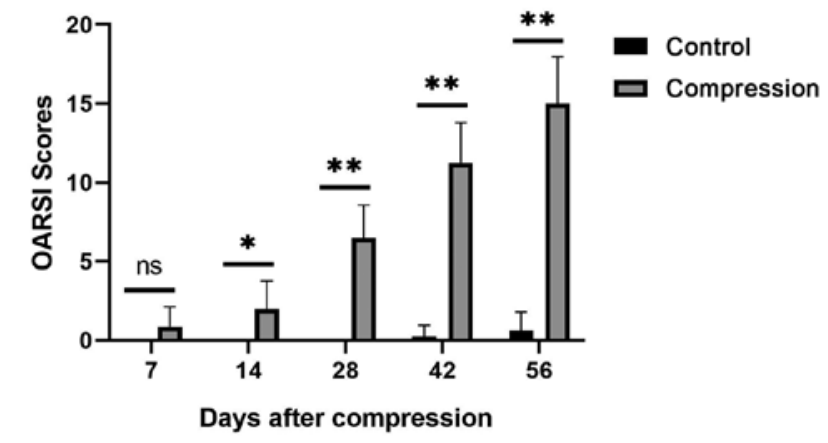

Figure 6. Comparison of OARSI scores of the facet joint between control and compression levels 7, 14, 28, 42 and 56 days after surgery. All data are presented as the mean \pm standard deviation $\left(n=8\right.$ per time point). ${ }^{*} \mathrm{P}<0.05$, ${ }^{* *} \mathrm{P}<0.01$. ns, not significant. OARSI, Osteoarthritis Research Society International.

Articular cartilage histological observation. Toluidine blue staining of the articular cartilage indicated smooth and normal surfaces without cracks, erosions or ulcerations in the control level. A smooth cartilage surface with normal cartilage thickness and regular chondrocyte arrangement were observed in control level at all time points selected ( 7 and 56 days following surgery) as shown in Fig. 5A and B. There was almost no difference between the two time points either in quantity or in the size of chondrocytes. Compared with the control level, the morphology of articular cartilage of the compression level was altered over time. At 7 days following surgery, the facet joint space narrowed (Fig. 5C), whereas at 14 days the cartilage surface became rough, with lighter staining on the surface (Fig. 5D). At 28 days, the number of chondrocytes in the surface layer were reduced, with a rougher cartilage surface and reduced thickness (Fig. 5E). At 42 days, the number of chondrocytes was further reduced and a further reduction in the thickness of the cartilage surface was observed (Fig. 5F). At 56 days following surgery, distinct roughness was identi- fied on the articular surface as well as a decrease in thickness (Fig. 5G).

Articular cartilage degeneration in the control and compression groups was evaluated according to the OARSI scoring system. The OARSI scoring results are presented in Fig. 6. Comparison between the control and compression groups was performed at the indicated days. Significant differences were observed at all the observed time points $(\mathrm{P}<0.05$ on day 14 and $\mathrm{P}<0.01$ for all other cases) apart from day $7(\mathrm{P}>0.05)$. These results demonstrated that facet joint articular cartilage degeneration in the compression level was significantly worse compared with that in the control level at all the observed time points, except for the first measured postoperative time point on day 7 .

Immunohistochemical staining. The immunohistochemical staining observations of IL- $1 \beta$ and TNF- $\alpha$ levels in the control group were almost the same in the indicated time points. Mild IL- $1 \beta$ and TNF- $\alpha$ staining was observed in the cartilage matrix (Figs. 7A and B as well as $8 \mathrm{~A}$ and $\mathrm{B}$ ). In the cartilage of the facet joint of the compression group, the percentage of IL-1 $\beta$ and TNF- $\alpha$-positive chondrocytes gradually increased from 7 to 56 days following surgery (Figs. $7 \mathrm{C}-\mathrm{G}$ and $8 \mathrm{C}-\mathrm{G}$ ). At 56 day after surgery, a high number of IL-1 $\beta$ and TNF- $\alpha$-positive chondrocytes was observed throughout the cartilage. Semi-quantitative analysis of immunohistochemical staining showed that expression of both IL-1 $\beta$ and TNF- $\alpha$ in the cartilage were significantly increased in the compression group in comparison with the control group at every time point (Tables I and II; P<0.01).

\section{Discussion}

Lumbar facet joint osteoarthritis is a common pathological condition during lumbar degeneration that is characterized by the presence of synovial inflammation, a narrow facet joint 
A

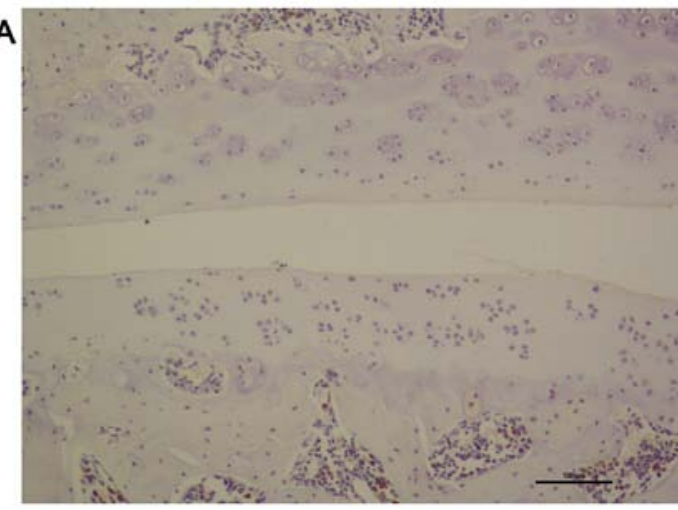

C

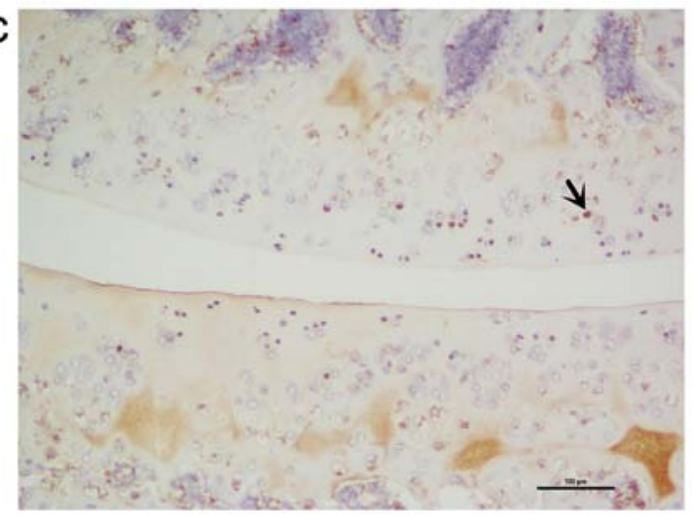

E

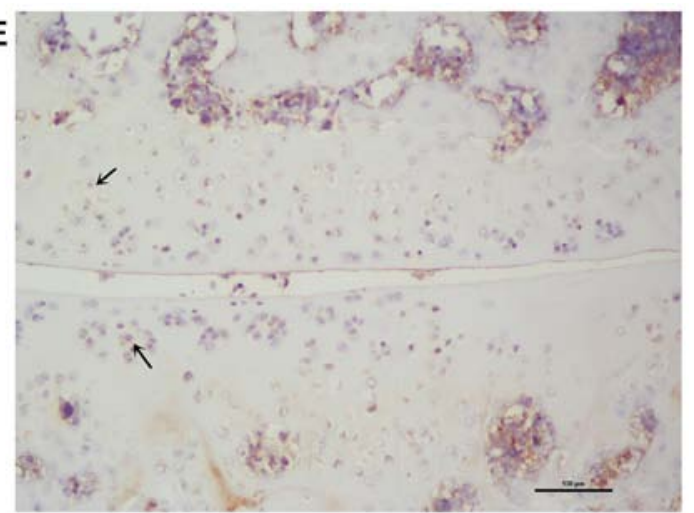

B

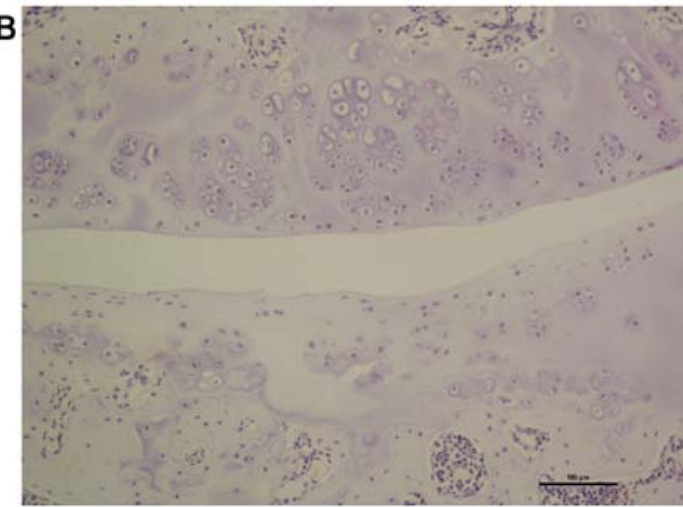

D

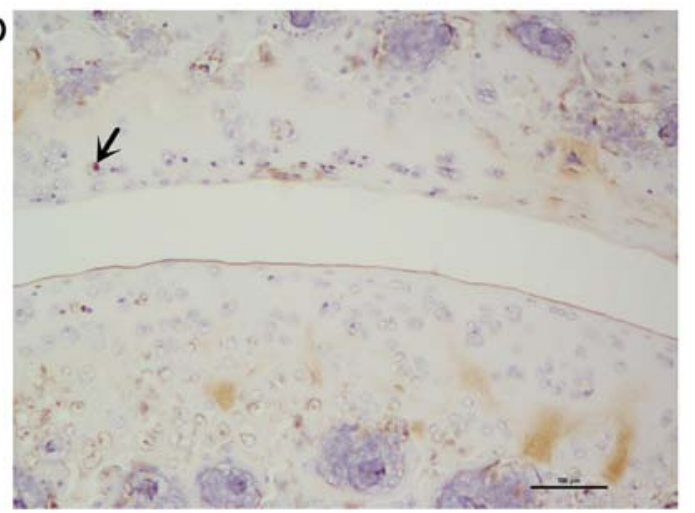

F

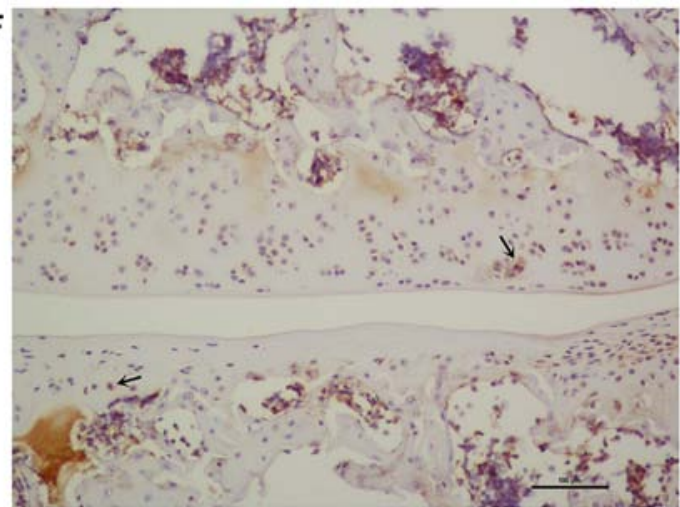

G

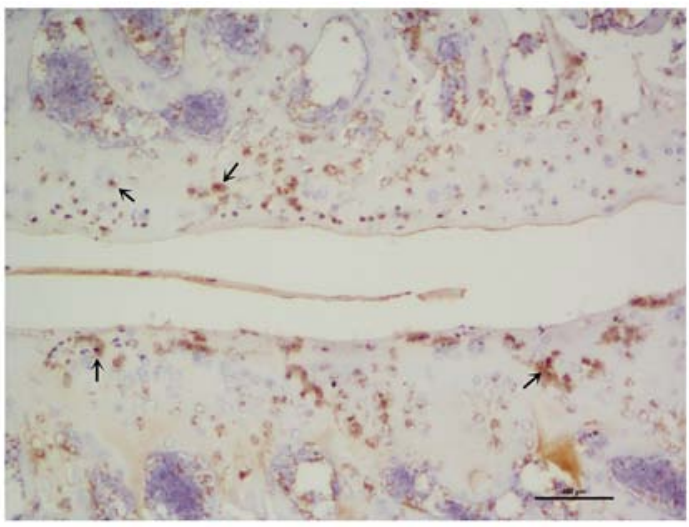

Figure 7. Representative immunohistochemical images showing IL-1 $\beta$ staining in the facet joint cartilage isolated from control and compression levels. Photomicrographs of the facet joint cartilage from the control levels at (A) 7 and (B) 56 days after surgery. Photomicrographs of the facet joint cartilage from the compression group at (C) 7, (D) 14, (E) 28, (F) 42 and (G) 56 days following surgery. The number of IL-1 $\beta$-positive chondrocytes (brown staining cells indicated by arrows) in the cartilage gradually increased from 7 to 56 days following surgery (magnification, x100; scale bar, $100 \mu \mathrm{m}$ ). IL, interleukin.

space and degeneration of the cartilage and the subchondral bone (14). This progressive degenerative change is symp- tomatic and may result in lower back pain (18). However, the mechanism of pathogenesis underlying lumbar facet 

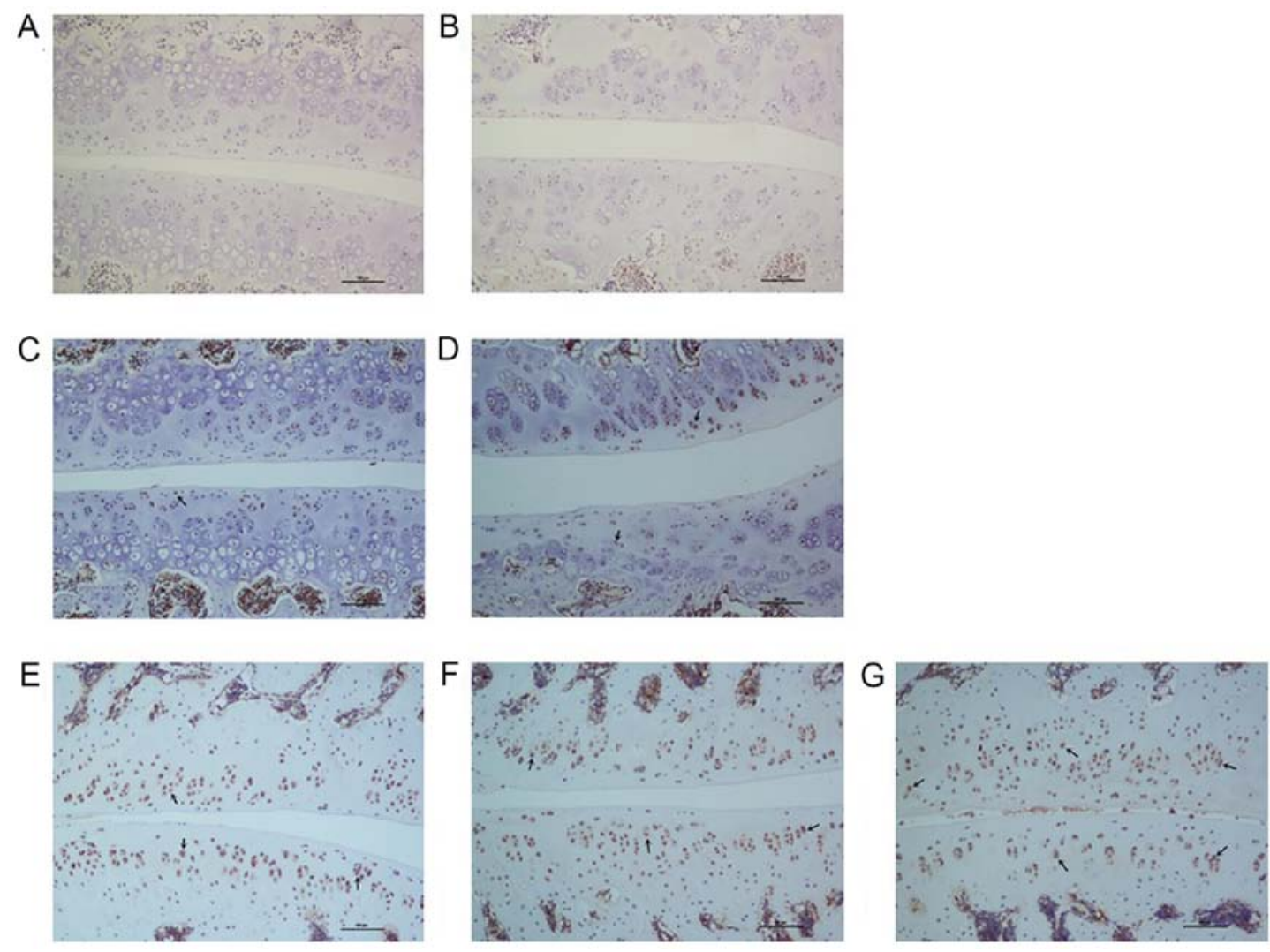

Figure 8. Representative immunohistochemical images showing TNF- $\alpha$ staining in the facet joint cartilage isolated from control and compression levels Photomicrographs of the facet joint cartilage from the control levels at (A) 7 and (B) 56 days after surgery. Photomicrographs of the facet joint cartilage from the compression group at (C) 7, (D) 14, (E) 28, (F) 42 and (G) 56 days following surgery. The number of TNF- $\alpha$-positive chondrocytes (brown staining cells indicated by arrows) in the cartilage gradually increased from 7 to 56 days following surgery (magnification, x100; Scale bar, $100 \mu \mathrm{m}$ ). TNF, tumor necrosis factor.

joint osteoarthritis remains unclear (19). Animal models can provide valuable insights into the mechanisms of action behind the symptomatic degeneration of the lower spine. To investigate the pathological mechanisms behind lumbar facet joint osteoarthritis further, in addition to discovering potentially novel prevention and treatment measures for this disease, the construction of an effective animal model is vital (20).

Until recently, two types of animal models have been established to simulate lumbar facet joint osteoarthritis, namely by chemical or mechanical induction (13). To the best of our knowledge, the majority of previous studies have focused on the chemically-induced animal models. In the chemically-induced animal models, different types of chemical agents are injected into the facet joints to induce autoimmune reactions, including collagenase, monosodium iodoacetate and complete Freund's adjuvant (21-26). The rapid onset of the autoimmune-induced inflammatory reaction is mediated by an intra-articular injection of exogenous chemical stimulators like the above in the facet joint. Chemically-induced animal models exhibit a rapid onset of facet joint osteoarthritis that is followed by the development of numerous cracks and defects in the cartilage $(22,26)$. In contrast, the corresponding pathological process noted in humans is reasonably slow with few cracks or defects. The aforementioned difference in the results is attributed to the different pathogenic mechanisms of action underlying facet joint osteoarthritis between humans and animals. Therefore, in contrast to human facet joint osteoar- thritis, the cause of which is associated with mechanical stress, chemically-induced animal models of osteoarthritis may be caused solely by chemical reactions.

Previous studies have demonstrated that compression on synovial joints results in pathological changes in osteoarthritis $(27,28)$. The lumbar facet joint exhibits similar pathological changes following compression injury. The human lumbar facet joint is burdened with axial compression force and shearing stress applied to the articular surface. Although normal amounts of loading stress provide the chondrocytes within facet joints with a natural nutritional supply for cell survival under physiological conditions, excessive stress loading on the facet joints can induce cartilage degeneration due to injury to the chondrocytes and matrix structure (28).

The first animal model of mechanically-induced facet joint pain was constructed by Henry et al (29) using a brief compression of the L5/6 facet joint. In this previously established novel rat model, following surgical exposure of the lumbar facet joints, modified clamps were applied for $3 \mathrm{~min}$ with an average 400 grams force to compress the spinal segments by $\sim 1 \mathrm{~mm}$. The end result was injury to the facet joint and increased pressure sensitivity from algometry at the operated area and mechanical allodynia at the hind paw. Unlike the chemically-induced models, this was a mechanically-induced model of lumbar facet joint osteoarthritis which simulated the pathology observed in humans during osteoarthritis more accurately. The disadvantage of this model may be the 
variation between the model's short-time compression and the human's chronic low back pain pathogenesis. To overcome this limitation, this present study used intraspinal compression springs that were fixed between spinous processes to provide a persistent force of compression. Since the compression springs were implanted between the spinous processes, the compression force line direction was parallel to the spinal axis and not to the facet joint. When the springs were elongated, lumbar facet joints experience stress from both axial compression force and shearing. The compression stress was a dynamic process, suggesting that the magnitude of the applied force varied according to the rat's lumbar position. These observations suggest that the animal model established in the present study presented higher accuracy and was more consistent with the human pathology $(30,31)$. In addition, this present study demonstrated that persistent mechanical compression injury of the facet joints in the rat model induced intra-articular osteoarthritis. This observation was substantiated by the considerable degradation observed in the facet joint cartilage compared with those noted in the cartilage of the control group. The pathological changes included synovitis, narrowing of the joint space, thinning of the cartilage and an increase in the expression of inflammatory factors. In addition, these changes appeared to be aggravated over time, suggesting a time-dependent association between the severity of osteoarthritis and compression injury in the facet joints.

The increased expression of the inflammatory cytokines, including TNF- $\alpha$ and notably IL-1 $\beta$, in the facet joint tissues of degenerative lumbar spinal disorders was first reported by Igarashi et al (32). This finding suggested that inflammatory cytokines in the degenerated facet joints may be associated with the cause of pain in degenerative lumbar disorders. In a number of previously established chemically-induced lumbar facet joint osteoarthritis animal models, increased expression levels of IL-1 $\beta$ and TNF- $\alpha$ were observed (22,24-26). Additionally, a strong correlation between the expression levels of inflammatory cytokines and facet joint degeneration-induced low back pain has also been previously demonstrated. These aforementioned findings were confirmed by the association between the expression levels of the inflammatory cytokines and the pain-associated behavioral changes $(29,33)$. In the present study, a time-dependent increase in IL- $1 \beta$ and TNF- $\alpha$ positive chondrocytes was also demonstrated as a result of the overloading of the lumbar facet joints. In contrast to this observation, late stage chemically-induced lumbar facet joint osteoarthritis animal models was accompanied with the induction of severe osteoarthritis, where the expression levels of the inflammatory cytokines were reduced due to mass chondrocyte apoptosis and cartilage matrix degradation (33). This observation was inconsistent with those of the present study, where the upregulation of inflammatory cytokine expression was detected. IL- $1 \beta$ and TNF- $\alpha$ serve key roles in synovitis and cartilage degeneration $(32,34,35)$. Therefore, the mechanically-induced lumbar facet joint osteoarthritis animal model presented in this present study appears to be more representative to the corresponding human disease compared with the chemically-induced models. Although chemical induction induces a rapid onset of severe osteoarthritis $(22,26)$, the pathological processes noted in these cases were considerably different compared with those noted in humans.
In conclusion, in the present study a novel rat model of lumbar facet joint osteoarthritis was successfully established by persistent mechanical compression injury. In addition, a time-dependent pathological process of osteoarthritis was confirmed as a result of facet joint overload, which stimulated the natural course of facet joint degeneration in humans more efficiently compared with other models such as chemically-induced and briefly mechanically-induced models. This present study can be used as a starting point for the investigation into the understanding and development of novel treatments for lower back pain associated with ailments in the lumbar facet joint.

\section{Acknowledgements}

Not applicable.

\section{Funding}

No funding was received.

\section{Availability of data and materials}

The datasets used and-or analyzed during the current study are available from the corresponding author on reasonable request.

\section{Authors' contributions}

YL and SH provided, conceived and designed the current study. YL and SP performed the experiments and analyzed the data. YL wrote, edited and reviewed the manuscript. All authors have read and approved the final manuscript.

\section{Ethics approval and consent to participate}

All experimental procedures were reviewed and approved by the Sixth Medical Center of the Chinese PLA General Hospital Animal Ethics Board (Beijing, China).

\section{Patient consent for publication}

Not applicable.

\section{Competing interests}

The authors declare that they have no competing interests.

\section{References}

1. Balagué F, Mannion AF, Pellisé F and Cedraschi C: Non-specific low back pain. Lancet 379: 482-491, 2012.

2. Hartvigsen J, Hancock MJ, Kongsted A, Louw Q, Ferreira ML, Genevay S, Hoy D, Karppinen J, Pransky G, Sieper J, et al; Lancet Low Back Pain Series Working Group: What low back pain is and why we need to pay attention. Lancet 391: 2356-2367, 2018.

3. Deyo RA and Weinstein JN: Low back pain. N Engl J Med 344: 363-370, 2001.

4. Langevin HM and Sherman KJ: Pathophysiological model for chronic low back pain integrating connective tissue and nervous system mechanisms. Med Hypotheses 68: 74-80, 2007.

5. Martin BI, Deyo RA, Mirza SK, Turner JA, Comstock BA, Hollingworth W and Sullivan SD: Expenditures and health status among adults with back and neck problems. JAMA 299: 656-664, 2008 
6. Nguyen C, Poiraudeau S and Rannou F: From Modic 1 vertebral-endplate subchondral bone signal changes detected by MRI to the concept of 'active discopathy'. Ann Rheum Dis 74: 1488-1494, 2015

7. Katz JN: Lumbar disc disorders and low-back pain: Socioeconomic factors and consequences. J Bone Joint Surg Am 88 (Suppl 2): 21-24, 2006.

8. Jensen MC, Brant-Zawadzki MN, Obuchowski N, Modic MT, Malkasian D and Ross JS: Magnetic resonance imaging of the lumbar spine in people without back pain. N Engl J Med 331: 69-73, 1994

9. Luoma K, Riihimäki H, Luukkonen R, Raininko R, Viikari-Juntura E and Lamminen A: Low back pain in relation to lumbar disc degeneration. Spine 25: 487-492, 2000.

10. Manchikanti L, Pampati V, Beyer C, Damron K and Barnhill RC: Evaluation of psychological status in chronic low back pain: Comparison with general population. Pain Physician 5: 149-155 2002.

11. Lyu Y, Chen $\mathrm{H}$ and Zheng ZM: Research progress of animal model of disc degeneration. Zhongguo Jizhu Jisui Zazhi 16 68-71, 2006.

12. Perolat R, Kastler A, Nicot B, Pellat JM, Tahon F, Attye A, Heck O, Boubagra K, Grand S and Krainik A: Facet joint syndrome: From diagnosis to interventional management. Insights Imaging 9: 773-789, 2018.

13. Gellhorn AC, Katz JN and Suri P: Osteoarthritis of the spine: The facet joints. Nat Rev Rheumatol 9: 216-224, 2013.

14. Lu Y and Hou S: Progress on the research of the low back pain animal model. Chin J Bone Jt 7: 146-149, 2018.

15. Sawa AG and Crawford NR: The use of surface strain data and a neural networks solution method to determine lumbar facet joint loads during in vitro spine testing. J Biomech 41: 2647-2653, 2008.

16. Pelletier JP, Jovanovic D, Fernandes JC, Manning P, Connor JR, Currie MG, Di Battista JA and Martel-Pelletier J: Reduced progression of experimental osteoarthritis in vivo by selective inhibition of inducible nitric oxide synthase. Arthritis Rheum 41: 1275-1286, 1998.

17. Pritzker KP, Gay S, Jimenez SA, Ostergaard K, Pelletier JP, Revell PA, Salter D and van den Berg WB: Osteoarthritis cartilage histopathology: Grading and staging. Osteoarthritis Cartilage 14: 13-29, 2006.

18. Boswell MV, Singh V, Staats PS and Hirsch JA: Accuracy of precision diagnostic blocks in the diagnosis of chronic spinal pain of facet or zygapophysial joint origin. Pain Physician 6: 449-456, 2003.

19. Kalichman L, Li L, Kim DH, Guermazi A, Berkin V, O'Donnell CJ, Hoffmann U, Cole R and Hunter DJ: Facet joint osteoarthritis and low back pain in the community-based population. Spine 33: 2560-2565, 2008.

20. Winkelstein BA: How can animal models inform on the transition to chronic symptoms in whiplash? Spine 36 (Suppl): S218-S225, 2011.

21. Yeh TT, Wu SS, Lee CH, Wen ZH, Lee HS, Yang Z, Nimni ME and Han B: The short-term therapeutic effect of recombinant human bone morphogenetic protein- 2 on collagenase-induced lumbar facet joint osteoarthritis in rats. Osteoarthritis Cartilage 15: 1357-1366, 2007
22. Yeh TT, Wen ZH, Lee HS, Lee CH, Yang Z, Jean YH, Wu SS, Nimni ME and Han B: Intra-articular injection of collagenase induced experimental osteoarthritis of the lumbar facet joint in rats. Eur Spine J 17: 734-742, 2008.

23. Adães S, Mendonça M, Santos TN, Castro-Lopes JM, Ferreira-Gomes $\mathbf{J}$ and Neto FL: Intra-articular injection of collagenase in the knee of rats as an alternative model to study nociception associated with osteoarthritis. Arthritis Res Ther 16: R10, 2014.

24. Kim JS, Kroin JS, Buvanendran A, Li X, van Wijnen AJ, Tuman KJ and Im HJ: Characterization of a new animal model for evaluation and treatment of back pain due to lumbar facet joint osteoarthritis. Arthritis Rheum 63: 2966-2973, 2011.

25. Gong K, Shao W, Chen H, Wang Z and Luo ZJ: Rat model of lumbar facet joint osteoarthritis associated with facet-mediated mechanical hyperalgesia induced by intra-articular injection of monosodium iodoacetate. J Formos Med Assoc 110: 145-152, 2011.

26. Shuang F, Zhu J, Song K, Hou S, Liu Y, Zhang C and Tang J: Establishment of a rat model of adjuvant-induced osteoarthritis of the lumbar facet joint. Cell Biochem Biophys 70: 1545-1551, 2014.

27. Kohatsu ND and Schurman DJ: Risk factors for the development of osteoarthrosis of the knee. Clin Orthop Relat Res 261: 242-246, 1990

28. Inoue N, Orías AAE and Segami K: Biomechanics of the lumbar facet joint. Spine Surg Relat Res 4: 1-7, 2019.

29. Henry JL, Yashpal K, Vernon H, Kim J and Im HJ: Lumbar facet joint compressive injury induces lasting changes in local structure, nociceptive scores, and inflammatory mediators in a novel rat model. Pain Res Treat 2012: 127636, 2012.

30. Varlotta GP, Lefkowitz TR, Schweitzer M, Errico TJ, Spivak J, Bendo JA and Rybak L: The lumbar facet joint: A review of current knowledge: Part 1: Anatomy, biomechanics, and grading. Skeletal Radiol 40: 13-23, 2011.

31. Rajeev A, Choudhry N, Shaikh M and Newby M: Lumbar facet joint septic arthritis presenting atypically as acute abdomen - A case report and review of the literature. Int J Surg Case Rep 25: 243-245, 2016.

32. Igarashi A, Kikuchi S, Konno S and Olmarker K: Inflammatory cytokines released from the facet joint tissue in degenerative lumbar spinal disorders. Spine 29: 2091-2095, 2004.

33. Shuang F, Hou SX, Zhu JL, Liu Y,Zhou Y, Zhang CL and Tang JG: Establishment of a rat model of lumbar facet joint osteoarthritis using intraarticular injection of urinary plasminogen activator. Sci Rep 5: 9828, 2015.

34. Apkarian AV, Lavarello S, Randolf A, Berra HH, Chialvo DR, Besedovsky HO and del Rey A: Expression of IL-1beta in supraspinal brain regions in rats with neuropathic pain. Neurosci Lett 407: 176-181, 2006

35. Tachihara H, Kikuchi S, Konno S and Sekiguchi M: Does facet joint inflammation induce radiculopathy?: An investigation using a rat model of lumbar facet joint inflammation. Spine 32: 406-412, 2007

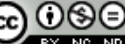

This work is licensed under a Creative Commons Attribution-NonCommercial-NoDerivatives 4.0 International (CC BY-NC-ND 4.0) License. 